\title{
STATISTICS OF WAVE FUNCTIONS FOR A POINT SCATTERER ON THE TORUS
}

\author{
ZEÉV RUDNICK AND HENRIK UEBERSCHÄR
}

\begin{abstract}
Quantum systems whose classical counterpart have ergodic dynamics are quantum ergodic in the sense that almost all eigenstates are uniformly distributed in phase space. In contrast, when the classical dynamics is integrable, there is concentration of eigenfunctions on invariant structures in phase space. In this paper we study eigenfunction statistics for the Laplacian perturbed by a delta-potential (also known as a point scatterer) on a flat torus, a popular model used to study the transition between integrability and chaos in quantum mechanics. The eigenfunctions of this operator consist of eigenfunctions of the Laplacian which vanish at the scatterer, and new, or perturbed, eigenfunctions. We show that almost all of the perturbed eigenfunctions are uniformly distributed in configuration space.
\end{abstract}

\section{INTRODUCTION}

Quantum systems whose classical counterpart have ergodic dynamics satisfy Schnirelman's theorem, which asserts that almost all eigenstates are uniformly distributed in phase space in an appropriate sense [21, 17, 23]. In contrast, when the classical dynamics is integrable, there is concentration of eigenfunctions on invariant structures in phase space. In this paper we study eigenfunction statistics for an intermediate system, that of a point scatterer on the flat torus.

The use of point scatterers, or $\delta$-potentials, goes back to the KronigPenney model [13] which is an idealized solvable model used to explain conductivity in a solid crystal and the appearance of electronic band structure. They have also been studied in the mathematical literature to explain the spurious occurrence of the Riemann zeros in a numerical experiment [8]. Billiards with a point scatterer have been used extensively in the quantum chaos literature, starting with Seba [19], to model quantum systems strongly perturbed in a region smaller than the wavelength of the particle.

The flat torus is a standard example of a system for which the geodesic flow is completely integrable. Placing a scatterer at a point $x_{0}$ in the torus does not change the classical dynamics except for a measure zero set of trajectories, and gives a quantum system whose dynamics is generated by an operator formally written as

$$
-\Delta+\alpha \delta_{x_{0}}
$$

Date: October 15, 2018. 
with $\delta_{x_{0}}$ being the Dirac mass at $x_{0}$ and $\alpha$ being a coupling parameter. Mathematically this corresponds to picking a self-adjoint extension of the Laplacian $-\Delta$ acting on functions vanishing near $x_{0}$ (see Section $\S 3$ and Appendix $\S$ A). Such extensions are parameterized by a phase $\phi \in(-\pi, \pi]$, with $\phi=\pi$ corresponding to the standard Laplacian $(\alpha=0$ in (1.1) $)$. We denote the corresponding operator by $-\Delta_{x_{0}, \phi}$, whose domain consists of a suitable space of functions $f(x)$ whose behavior near $x_{0}$ is given by

$$
f(x)=C\left(\cos \frac{\phi}{2} \cdot \frac{\log \left|x-x_{0}\right|}{2 \pi}+\sin \frac{\phi}{2}\right)+o(1), \quad x \rightarrow x_{0}
$$

for some constant $C$. For $\phi=\pi$ the eigenvalues are those of the standard Laplacian. For $\phi \neq \pi(\alpha \neq 0)$ the resulting spectral problem still has the eigenvalues from the unperturbed problem, with multiplicity decreased by one, as well as a new set $\Lambda_{\phi}$ of eigenvalues interlaced between the sequence of unperturbed eigenvalues, each appearing with multiplicity one, and satisfying the spectral equation

$$
\sum_{n}\left|\psi_{n}\left(x_{0}\right)\right|^{2}\left(\frac{1}{\lambda_{n}-\lambda}-\frac{\lambda_{n}}{\lambda_{n}^{2}+1}\right)=c_{0} \tan \frac{\phi}{2}
$$

for a certain $c_{0}>0$, where $\left\{\psi_{n}(x)\right\}$ form an orthonormal basis of eigenfunctions for the unperturbed problem: $-\Delta \psi_{n}=\lambda_{n} \psi_{n}$. The eigenfunction corresponding to $\lambda \in \Lambda_{\phi}$ is the Green's function

$$
G_{\lambda}\left(x ; x_{0}\right)=(\Delta+\lambda)^{-1} \delta_{x_{0}} .
$$

Our main result is that for almost all $\lambda \in \Lambda_{\phi}$, the perturbed eigenfunctions $G_{\lambda}\left(\bullet ; x_{0}\right)$ are uniformly distributed in position space. To formulate the result precisely, we denote by

$$
g_{\lambda}(x):=\frac{G_{\lambda}\left(x ; x_{0}\right)}{\left\|G_{\lambda}\right\|_{2}}
$$

the $L^{2}$-normalized Green's function:

Theorem 1.1. Fix $\phi \in(-\pi, \pi)$. There is a subset $\Lambda_{\phi, \infty} \subset \Lambda_{\phi}$ of density one so that for all observables $a \in C^{\infty}\left(\mathbb{T}^{2}\right)$,

$$
\int_{\mathbb{T}^{2}} a(x) g_{\lambda}(x)^{2} d x \rightarrow \frac{1}{\operatorname{area}\left(\mathbb{T}^{2}\right)} \int_{\mathbb{T}^{2}} a(x) d x
$$

as $\lambda \rightarrow \infty$ along the subsequence $\Lambda_{\phi, \infty}$

\section{Remarks:}

For the eigenfunctions of the unperturbed Laplacian, there is a variety of possible limits in the position representation, which were investigated by Jakobson [10].

A result of the same nature as our Theorem 1.1 was recently obtained in [15] for billiards in rational polygons. There it is shown that for any orthonormal basis of eigenfunctions, there is a density one subsequence which equidistributes in configuration space. The method of [15] adapts the proof 
of quantum ergodicity for billiards of [24] to work in configuration space and inputs the theorem of Kerckhoff, Masur and Smillie [12] who showed that for rational polygons, the billiard flow is uniquely ergodic in almost every direction. Our argument here is completely different and is very specific to this particular model.

A related, and in some sense complementary, issue was studied by Berkolaiko, Keating and Winn [2] who predict that for an irrational torus with a point scatterer there is a subsequence of eigenfuctions which "scar" in momentum space, and this was proved by Keating, Marklof and Winn [11] to be the case assuming that the eigenvalues of the Laplacian on the unperturbed irrational torus have Poisson spacing distribution, as is predicted by the Berry-Tabor conjecture.

It is important to note that we (as well as [2, 11]) deal with the limit of large energy $\lambda \rightarrow \infty$ for a fixed phase $\phi \neq \pi$, which is called the weak coupling limit in the physics literature. An interesting problem would be to understand the strong coupling limit, where $\lambda \rightarrow \infty$ together with $\phi \rightarrow \pi$ while $\tan (\phi / 2) \approx \log \lambda$, so that the RHS of the spectral equation (1.3) blows up. In that range it has been argued that the spectrum displays intermediate statistics [20, 5, 3, 4, 17].

Acknowledgments: We thank Maja Rudolph for her help with the numerical investigation of some of these issues, and John Friedlander for discussions concerning sums of two squares. Z.R. was partially supported by the Israel Science Foundation (grant No. 1083/10). H.U. was supported by a Minerva Fellowship.

\section{THE FLAT TORUS}

2.1. Basic setup. We consider a flat torus $\mathbb{T}^{2}$ obtained by identifying opposite sides of a rectangle with side lengths $2 \pi / a, 2 \pi a$, so that $\mathbb{T}^{2}=\mathbb{R}^{2} / 2 \pi \mathcal{L}_{0}$ where $\mathcal{L}_{0}=\mathbb{Z}(1 / a, 0) \oplus \mathbb{Z}(0, a)$ is a unimodular lattice.

An orthonormal basis of eigenfunctions for the Laplacian $\Delta$ on $\mathbb{T}^{2}$ consists of the exponentials

$$
\frac{1}{2 \pi} e^{i\langle x, \xi\rangle}
$$

where $\xi$ ranges over the dual, or reciprocal, lattice

$$
\mathcal{L}=\left\{x \in \mathbb{R}^{2}:\langle x, \ell\rangle \in \mathbb{Z}, \quad \forall \ell \in \mathcal{L}_{0}\right\}=\left\{\left(m a, \frac{n}{a}\right): m, n \in \mathbb{Z}\right\}
$$

The eigenvalues of the Laplacian on $\mathbb{T}^{2}$ are the norms $|\xi|^{2}$ of the vectors of the dual lattice $\mathcal{L}$. Weyl's law for the torus, establishing the asymptotics of the counting function $N(x)$ of eigenvalues below $x$, is equivalent to counting the number of points of the lattice $\mathcal{L}$ in a disk (equivalently the number of points of the standard lattice $\mathbb{Z}^{2}$ in an ellipse), and therefore reads

$$
N(x)=\#\left\{|\xi|^{2} \leq x: \xi \in \mathcal{L}\right\}=\pi x+O\left(x^{\theta}\right)
$$


The exponent $\theta$ in the remainder term is known to be at least $\theta>1 / 4$. The "trivial" bound on the remainder term, as the length of the boundary, translates into $\theta \leq 1 / 2$. A nontrivial bound uses Poisson summation and the method of stationary phase leads to $\theta \leq 1 / 3$. We will need a better bound

$$
\theta<\frac{1}{3}
$$

such as the one due to van der Corput [22]. The current world record of $\theta \leq 131 / 416+o(1)$ is due to Huxley [9].

Using the remainder term (2.3), we may deduce a bound for the number of lattice points in an annulus: Define

$$
A(\lambda, L)=\left\{\xi \in \mathcal{L}: \lambda-L<|\xi|^{2}<\lambda+L\right\}
$$

Then (2.3) implies

$$
\# A(\lambda, L)=2 \pi L+O\left(\lambda^{\theta}\right)
$$

2.2. Multiplicities. Denote by $\mathcal{N}=\left\{0<n_{1}<\ldots\right\}$ the set of norms of the dual lattice vectors. The multiplicities in the spectrum are

$$
r_{\mathcal{L}}(n)=\#\left\{\xi \in \mathcal{L}:|\xi|^{2}=n\right\}
$$

The lattice $\mathcal{L}$ is rational if, after a suitable scaling, the norms $|\xi|^{2}$ are all rational. The norm of an arbitrary lattice vector $\xi=(m a, n / a)$ is

$$
|\xi|^{2}=a^{2} m^{2}+n^{2} / a^{2}=\frac{1}{a^{2}}\left(a^{4} m^{2}+n^{2}\right)
$$

so that the lattice is rational if and only if $a^{4} \in \mathbb{Q}$ is rational.

In the irrational case, the multiplicities are entirely due to the reflection symmetries $(x, y) \mapsto( \pm x, \pm y)$ and are given by

$$
r_{\mathcal{L}}(n)= \begin{cases}1, & n=0 \\ 2, & n=a^{2} u^{2} \text { or } v^{2} / a^{2}, u, v \in \mathbb{Z} \\ 4, & \text { otherwise }\end{cases}
$$

and in particular are generically equal to 4 .

For the rational case the multiplicities are complicated arithmetic functions. For instance in the case of the standard lattice $\mathcal{L}=\mathbb{Z}^{2}$, the multiplicity $r(n)=\#\left\{(x, y) \in \mathbb{Z}^{2}: x^{2}+y^{2}=n\right\}$ depends on the prime factorization of the integer $n$. In any case, it is well known that we have an upper bound on the multiplicities of the form (see e.g. the proof of [16, Lemma 7.2])

$$
r_{\mathcal{L}}(n) \ll n^{o(1)}
$$

The counting function of the norms is

$$
\mathcal{N}(x):=\#\{n \in \mathcal{N}: n \leq x\}
$$


Since we have the upper bound (2.10) and since we know that the sum over $n \leq x$ of the multiplicities is asymptotically $\pi x(\underline{2.3})$, we deduce a lower bound

$$
\mathcal{N}(x) \gg x^{1-o(1)}
$$

2.3. Nearest neighbor gaps. If the norms are ordered by $\mathcal{N}=\left\{0<n_{1}<\right.$ $\left.n_{2}<\ldots\right\}$, we need to understand the spacings (or gaps) $n_{k+1}-n_{k}$ between successive norms. The individual values are difficult to understand. From (2.3) we certainly have $n_{k+1}-n_{k} \ll n_{k}^{\theta}$. However one can do better by arguing as follows [1]: First find the largest integer square $u^{2}<n_{k+1} / a^{2}$, which one can do so that $n_{k+1}-a^{2} u^{2} \ll \sqrt{n_{k+1}}$. After that find the largest square $v^{2} / a^{2}<n_{k+1}-a^{2} u^{2}$, which one can do so that $n_{k+1}-a^{2} u^{2}-v^{2} / a^{2} \ll$ $\sqrt{n_{k+1}-a^{2} u^{2}} \ll n_{k+1}^{1 / 4}$. Thus we found a norm $n=a^{2} u^{2}+v^{2} / a^{2} \in \mathcal{N}$ with $n<n_{k+1}$ so that $n \leq n_{k}$ giving

$$
n_{k+1}-n_{k} \ll n_{k}^{1 / 4}
$$

The average spacing for norms up to $x$ is, using (2.13),

$$
\frac{1}{\mathcal{N}(x)} \sum_{n_{k} \leq x}\left(n_{k}-n_{k-1}\right)=\frac{x(1+o(1))}{\mathcal{N}(x)}
$$

and by the lower bound (2.12) we deduce that

$$
\frac{1}{\mathcal{N}(x)} \sum_{n_{k} \leq x}\left(n_{k}-n_{k-1}\right) \ll x^{o(1)}
$$

Since we are dealing with averages of positive quantities, we find:

Lemma 2.1. For almost all $k$, that is on a density one sequence, the spacings satisfy

$$
n_{k+1}-n_{k} \ll n_{k}^{o(1)}
$$

\section{POINT SCATTERERS AND $\delta$-POTENTIALS}

In this section we review the theory of point scatterers (see [6]), with some details left to Appendix A.

3.1. A finite-dimensional model. We want to study the Schrödinger operator with a delta-potential on the flat $D$-dimensional torus $\mathbb{T}^{D}=\mathbb{R}^{D} / 2 \pi \mathcal{L}_{0}$, where $\mathcal{L}_{0} \subset \mathbb{R}^{D}$ is a unimodular lattice. The operator is formally given by

$$
-\Delta+\alpha \delta
$$

where $\delta$ is the Dirac delta-function at the point $x_{0}$.

To make sense of the operator (3.1), we say that for a wave function $\psi \in C^{\infty}\left(\mathbb{T}^{D}\right)$, multiplication by $\delta$ should give

$$
\delta \psi=\psi\left(x_{0}\right) \delta=\langle\psi, \delta\rangle \delta
$$

which we try to think of as a rank-one operator. 
As an approximation, it is useful to examine a finite-dimensional model: a rank one perturbation of a self-adjoint operator $H_{0}$ on a finite-dimensional Hilbert space $\mathcal{H}$ of the form

$$
H=H_{0}+\alpha P_{v}, \quad P_{v} u=\langle u, v\rangle v, \quad u \in \mathcal{H}
$$

where $0 \neq v \in \mathcal{H}$ and $\alpha \neq 0$ is real. Let $\phi_{n}$ be an orthonormal basis of $\mathcal{H}$ consisting of eigenvectors $H_{0}$, with eigenvalues $\epsilon_{n}: H_{0} \phi_{n}=\epsilon_{n} \phi_{n}$. The eigenvectors $\phi_{n}$ of $H_{0}$ with $\left\langle\phi_{n}, v\right\rangle=0$ are clearly still eigenvectors of $H$. The new part of the spectrum is given by:

Lemma 3.1. The eigenvalues $E \notin$ Spec $\left(H_{0}\right)$ of the perturbed operator $H$ are the solutions of the equation

$$
\left\langle\left(E-H_{0}\right)^{-1} v, v\right\rangle=\frac{1}{\alpha}
$$

or equivalently

$$
\sum_{n} \frac{\left|\left\langle v, \phi_{n}\right\rangle\right|^{2}}{E-\epsilon_{n}}=\frac{1}{\alpha}
$$

with corresponding eigenfunction

$$
u=\left(E-H_{0}\right)^{-1} v
$$

Proof. We rewrite the eigenvalue equation $H u=E u$ for $H$ in the form

$$
\left(E-H_{0}\right) u=\alpha\langle u, v\rangle v
$$

If $E \notin \operatorname{Spec}\left(H_{0}\right)$ then necessarily $\langle u, v\rangle \neq 0$ and we find that

$$
u=\alpha\langle u, v\rangle\left(E-H_{0}\right)^{-1} v
$$

Thus up to a scalar multiple

$$
u=\left(E-H_{0}\right)^{-1} v
$$

Substituting (3.9) in the eigenvalue equation (3.7) gives

$$
v=\alpha\left\langle\left(E-H_{0}\right)^{-1} v, v\right\rangle v
$$

that is

$$
\left\langle\left(E-H_{0}\right)^{-1} v, v\right\rangle=\frac{1}{\alpha}
$$

Expanding $v=\sum_{n}\left\langle v, \phi_{n}\right\rangle \phi_{n}$ in terms of the normalized eigenvectors $\phi_{n}$ of $H_{0}$ gives

$$
\sum_{n} \frac{\left|\left\langle v, \phi_{n}\right\rangle\right|^{2}}{E-\epsilon_{n}}=\frac{1}{\alpha}
$$

Conversely, if $E \notin \operatorname{Spec}\left(H_{0}\right)$ and (3.4) holds, take $u=\left(E-H_{0}\right)^{-1} v$ as in (3.6). Then

$$
(H-E) u=\left(H_{0}-E\right) u+\alpha\langle u, v\rangle v
$$

Since $\left(H_{0}-E\right) u=\left(H_{0}-E\right)\left(E-H_{0}\right)^{-1} v=-v$ and $\alpha\langle u, v\rangle v=\alpha\langle(E-$ $\left.\left.H_{0}\right)^{-1} v, v\right\rangle v=v$ by (3.4) we find that $H u=E u$. 
Now take for $H_{0}$ the free Schrödinger operator $H_{0}=-\Delta$ acting on $C^{\infty}\left(\mathbb{T}^{D}\right) \subset L^{2}\left(\mathbb{T}^{D}\right)$, and $v=\delta_{x_{0}}$. Then the eigenfunctions (3.6) with eigenvalues $E \notin \operatorname{Spec}\left(H_{0}\right)$ are the Green's function

$$
G_{E}\left(\bullet ; x_{0}\right)=\left(E-H_{0}\right)^{-1} \delta_{x_{0}}
$$

However the sum (3.5) diverges; indeed,in that case the RHS of (3.5) equals

$$
\sum_{n} \frac{\left|\phi_{n}\left(x_{0}\right)\right|^{2}}{E-\epsilon_{n}}
$$

which is divergent in dimension $D>1$, by Weyl's law. In fact (3.14) is just the Green's function evaluated on the diagonal, which is divergent in dimension $D>1$. Thus one needs a regularization procedure.

3.2. Regularization. One regularization procedure is through the theory of self-adjoint extensions. A succinct account of this procedure is given in [6]. For the reader's convenience this will be reviewed in Appendix $\S$ A, One starts with the standard Laplacian $\Delta$, for which an orthogonal basis of eigenfunctions are the exponentials $e^{i\langle\xi, x\rangle}, \xi$ in the dual lattice $\mathcal{L}$ with corresponding eigenvalue $|\xi|^{2}$. The idea is that for functions vanishing at the point $x_{0}$ all candidates have to coincide with the unperturbed operator $H_{0}=-\Delta$. We want to extend it to a bigger space. Denoting by $-\Delta_{x_{0}}$ the unperturbed operator restricted to $C_{c}^{\infty}\left(\mathbb{T}^{D} \backslash\left\{x_{0}\right\}\right)$, one finds that the adjoint has as its domain $\operatorname{Dom}\left(-\Delta_{x_{0}}^{*}\right)$ the Sobolev space $H^{2}\left(\mathbb{T}^{D} \backslash\left\{x_{0}\right\}\right)$. In dimension $D=2,3$ this equals 11 the space of $f \in L^{2}\left(\mathbb{T}^{D}\right)$ for which $\exists A \in \mathbb{C}$ s.t.

$$
\Delta f-A \delta_{x_{0}} \in L^{2}\left(\mathbb{T}^{D}\right)
$$

and for such $f$, there is some $B \in \mathbb{C}$ so that for $x$ near $x_{0}$,

$$
f(x)=A G^{(D)}\left(\left|x-x_{0}\right|\right)+B+o(1),
$$

where

$$
G^{(D)}(r)=\left\{\begin{array}{ll}
\frac{1}{2 \pi} \log r, & D=2 \\
-\frac{1}{4 \pi r}, & D=3
\end{array} .\right.
$$

One finds that in dimension $D=2,3$ there is a one-parameter family of extensions, parameterized by $\phi \in \mathbb{R} / 2 \pi \mathbb{Z} \simeq U(1)$, denoted by $-\Delta_{\phi, x_{0}}$ with domain given by $f \in \operatorname{Dom}\left(-\Delta_{x_{0}}^{*}\right)$ for which there is some $a \in \mathbb{C}$ with

$$
f(x)=a\left(\cos \frac{\phi}{2} \cdot G^{(D)}\left(\left|x-x_{0}\right|\right)+\sin \frac{\phi}{2}\right)+o(1), \quad x \rightarrow x_{0}
$$

The action of $-\Delta_{\phi, x_{0}}$ on $f$ satisfying (3.15) is then given by

$$
-\Delta_{\phi, x_{0}} f=-\Delta f+A \delta_{x_{0}}
$$

\footnotetext{
${ }^{1}$ In dimension $D=1$ one wants $\Delta f-c_{0} \delta-c_{1} \delta^{\prime} \in L^{2}\left(\mathbb{T}^{1}\right)$, while in dimensions $D \geq 4$, $H_{0}$ is essentially self adjoint and there are no self-adjoint extensions.
} 
The divergent equation (3.5) is replaced by the convergent equation for the new eigenvalues $\lambda \notin \sigma(\Delta)$ :

$$
\sum_{\xi \in \mathcal{L}}\left\{\frac{1}{|\xi|^{2}-\lambda}-\frac{|\xi|^{2}}{|\xi|^{4}+1}\right\}=c_{0} \tan \frac{\phi}{2}
$$

where

$$
c_{0}=\sum_{\xi \in \mathcal{L}} \frac{1}{|\xi|^{4}+1}
$$

We can rewrite (3.20) as

$$
\sum_{n \in \mathcal{N}} r_{\mathcal{L}}(n)\left\{\frac{1}{n-\lambda}-\frac{n}{n^{2}+1}\right\}=c_{0} \tan \frac{\phi}{2}
$$

where

$$
r_{\mathcal{L}}(n)=\#\left\{n=|\xi|^{2}: \xi \in \mathcal{L}\right\}
$$

and $\mathcal{N}$ is the set of norms of vectors in $\mathcal{L}$ (without multiplicity). The corresponding eigenfunction is a multiple of $G_{\lambda}\left(x ; x_{0}\right)=(\Delta+\lambda)^{-1} \delta_{x_{0}}$, which has the $L^{2}$-expansion

$$
G_{\lambda}\left(x, x_{0}\right)=-\frac{1}{4 \pi^{2}} \sum_{\xi \in \mathcal{L}} \frac{\exp \left(\mathrm{i} \xi \cdot\left(x-x_{0}\right)\right)}{|\xi|^{2}-\lambda}, \quad x \neq x_{0} .
$$

As may be seen from (3.21), the new eigenvalues interlace between the sequence $\mathcal{N}=\left\{n_{1}<n_{2}<\ldots\right\}$ of norms, see Figure 1, We may thus label the perturbed eigenvalues $\lambda_{k}=\lambda_{k}^{\phi}$ so that

$$
n_{1}<\lambda_{1}<n_{2}<\lambda_{2}<\cdots<n_{k}<\lambda_{k}<n_{k+1}<\ldots
$$

3.3. The density of states. The density of states of the perturbed eigenvalues depends strongly on the particular torus, that is on the lattice it determines.

For the standard lattice $\mathbb{Z}^{2}$, a theorem of Landau [14] asserts that

$$
\#\{n \in \mathcal{N}, n \leq x\} \sim B \frac{x}{\sqrt{\log x}}
$$

where $B=\frac{1}{\sqrt{2}} \prod\left(1-p^{-2}\right)^{-1 / 2}=0.764 \ldots$, the product over primes $p=$ $3 \bmod 4$. Consequently we deduce a form of Weyl's law for the perturbed spectrum $\Lambda_{\phi}$ of $\Delta_{\phi, x_{0}}$ for the standard lattice:

$$
\#\left\{\lambda \in \Lambda_{\phi}: \lambda \leq x\right\} \sim B \frac{x}{\sqrt{\log x}}
$$

In the irrational case, the multiplicities are typically 4 , see (2.9). Then Weyl's law in those cases would read as

$$
\#\left\{\lambda \in \Lambda_{\phi}: \lambda \leq x\right\} \sim \frac{\pi}{4} x
$$




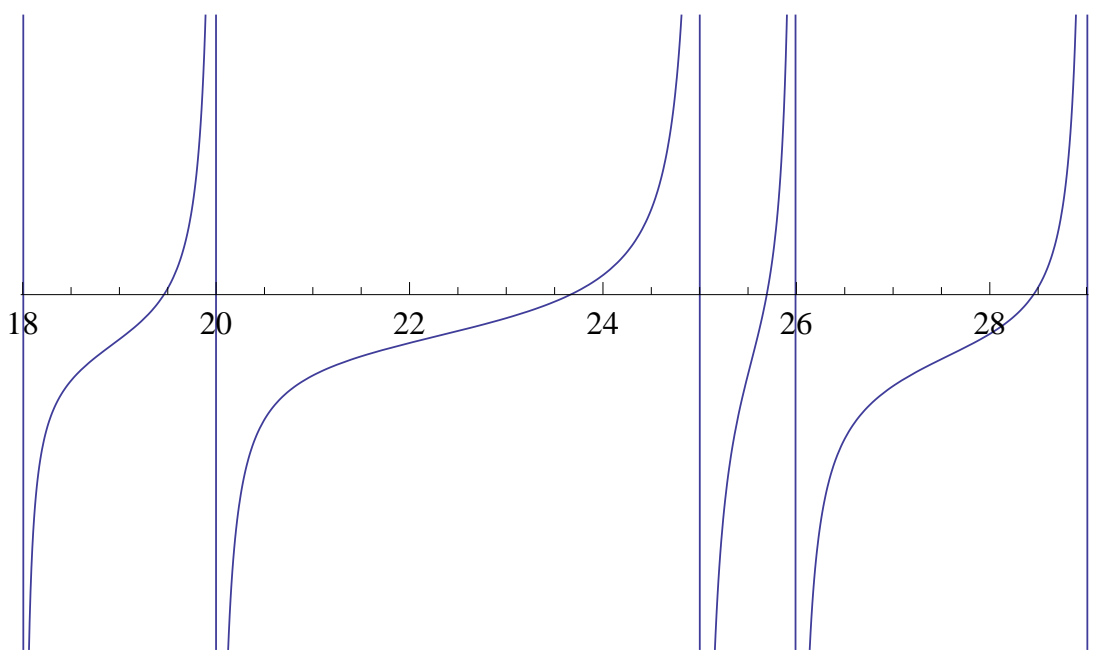

FiguRE 1. A plot of the spectral function on the LHS of the eigenvalue equation (3.21) for the standard lattice $\mathcal{L}=\mathbb{Z}^{2}$. The intersections of the plot with the $x$-axis are the perturbed eigenvalues corresponding to $\phi=0$, alternating with the norms.

\section{ThE NORM OF $G_{\lambda}$}

We will need a lower bound on the $L^{2}$-norm of the Green's function $G_{\lambda}$. We are able to get a good bound for a sub-sequence of density one. To define this subsequence, we recall our discussion of the gaps between consecutive norms.

According to Lemma 2.1, for almost all $k$ we have

$$
n_{k+1}-n_{k} \ll n_{k}^{o(1)}
$$

We define the set $\Lambda_{g} \subset \Lambda$ of eigenvalues $\lambda_{k}$ (using the labeling (3.23) ) so that (4.1) holds:

$$
\Lambda_{g}=\left\{\lambda_{k} \in \Lambda: n_{k+1}-n_{k} \ll \lambda_{k}^{o(1)}\right\}
$$

By the discussion above, this is a set of density one in $\Lambda$ (and conjecturally all of $\Lambda$ ).

Lemma 4.1. For $\lambda \in \Lambda_{g}$ (i.e. for almost all $\lambda$ ), we have

$$
\left\|G_{\lambda}\right\| \gg \frac{1}{\lambda^{o(1)}}
$$

Proof. Let $n_{k}=n_{k}(\lambda), n_{k+1}=n_{k+1}(\lambda)$ be consecutive norms so that $n_{k}<$ $\lambda<n_{k+1}$. Then trivially

$$
\left\|G_{\lambda}\right\|_{2}^{2} \gg \sum_{n \in \mathcal{N}} \frac{r_{\mathcal{L}}(n)}{(n-\lambda)^{2}} \geq \frac{1}{\left(n_{k}-\lambda\right)^{2}}>\frac{1}{\left(n_{k+1}-n_{k}\right)^{2}}
$$


Since for $\lambda \in \Lambda_{g}$ we know that $n_{k+1}-n_{k} \ll n_{k}^{o(1)}$, (4.3) follows.

It is natural to conjecture that (4.3) holds for all $\lambda$.

\section{TRunCATION}

For $L>0$ let $A(\lambda, L)$ be the set of lattice points in the annulus $\lambda-L<$ $|x|^{2}<\lambda+L$ :

$$
A(\lambda, L)=\left\{\xi \in \mathcal{L}:\left.|| \xi\right|^{2}-\lambda \mid<L\right\}
$$

We denote the truncated Green's function by

$$
G_{\lambda, L}\left(x, x_{0}\right)=-\frac{1}{4 \pi^{2}} \sum_{\xi \in A(\lambda, L)} \frac{\exp \left(\mathrm{i} \xi \cdot\left(x-x_{0}\right)\right)}{|\xi|^{2}-\lambda} .
$$

Let $g_{\lambda}$ and $g_{\lambda, L}$ be the $L^{2}$-normalized Green's function and its truncation:

$$
g_{\lambda}=\frac{G_{\lambda}}{\left\|G_{\lambda}\right\|}, \quad g_{\lambda, L}=\frac{G_{\lambda, L}}{\left\|G_{\lambda, L}\right\|}
$$

We have the following approximation.

Lemma 5.1. Let $L=\lambda^{\delta}, \theta / 2<\delta<1$. As $\lambda \rightarrow \infty$ along $\Lambda_{g}$,

$$
\left\|g_{\lambda}-g_{\lambda, L}\right\|_{2} \rightarrow 0
$$

Proof. Note that

$$
\begin{aligned}
\left\|\frac{G_{\lambda}}{\left\|G_{\lambda}\right\|_{2}}-\frac{G_{\lambda, L}}{\left\|G_{\lambda, L}\right\|_{2}}\right\|_{2} \leq & \frac{\left\|G_{\lambda}-G_{\lambda, L}\right\|_{2}}{\left\|G_{\lambda}\right\|_{2}} \\
& +\left\|G_{\lambda, L}\right\|_{2}\left|\frac{1}{\left\|G_{\lambda}\right\|_{2}}-\frac{1}{\left\|G_{\lambda, L}\right\|_{2}}\right| \\
\leq & 2 \frac{\left\|G_{\lambda}-G_{\lambda, L}\right\|_{2}}{\left\|G_{\lambda}\right\|_{2}} .
\end{aligned}
$$

We have

$$
\left\|G_{\lambda}-G_{\lambda, L}\right\|_{2}^{2}=\frac{1}{16 \pi^{4}} \sum_{\left.|| \xi\right|^{2}-\lambda \mid \geq \lambda^{\delta}} \frac{1}{\left(|\xi|^{2}-\lambda\right)^{2}}
$$

We recall how to evaluate lattice sums using summation by parts:

Let $n_{1}<n_{2}<\ldots$ be the set of norms, and

$$
N(t)=\sum_{n_{k} \leq t} r_{\mathcal{L}}\left(n_{k}\right)
$$

Then for a smooth function $f(t)$ on $\mathbb{R}$ we have

$$
\sum_{n_{A}<|\xi|^{2} \leq n_{B}} f\left(|\xi|^{2}\right)=N\left(n_{B}\right) f\left(n_{B}\right)-N\left(n_{A}\right) f\left(n_{A+1}\right)-\int_{n_{A+1}}^{n_{B}} f^{\prime}(t) N(t) d t
$$


Now use the lattice count with remainder (2.3) to get

$$
\begin{aligned}
\sum_{n_{A}<|\xi|^{2} \leq n_{B}} f\left(|\xi|^{2}\right) & =\pi \int_{n_{A+1}}^{n_{B}} f(t) d t \\
+ & O\left(n_{B}^{\theta} f\left(n_{B}\right)+n_{A+1}^{\theta} f\left(n_{A}\right)\right)+O\left(\int_{n_{A+1}}^{n_{B}}\left|f^{\prime}(t)\right| t^{\theta} d t\right)
\end{aligned}
$$

Applying (5.8) with $f(t)=1 /(t-\lambda)^{2}$, once with $n_{A}=n_{1}$ and $n_{B} \leq$ $\lambda-L<n_{B+1}$ and then with $n_{A-1}<\lambda+L \leq n_{A}<n_{A+1}$ and $n_{B}=\infty$ gives

$$
\left\|G_{\lambda}-G_{\lambda, L}\right\|_{2}^{2} \ll \frac{1}{L}+\frac{\lambda^{\theta}}{L^{2}}
$$

Since for $\lambda \in \Lambda_{g}$ we have $\left\|G_{\lambda}\right\|^{2} \gg 1 / \lambda^{o(1)}$ by Lemma 4.1, we find

$$
\frac{\left\|G_{\lambda}-G_{\lambda}^{L}\right\|_{2}^{2}}{\left\|G_{\lambda}\right\|_{2}^{2}} \ll \lambda^{o(1)}\left(\frac{1}{L}+\frac{\lambda^{\theta}}{L^{2}}\right)
$$

which tends to zero if $\delta>\theta / 2$.

Consequently we may study the matrix elements by replacing $g_{\lambda}$ by the truncated version $g_{\lambda, L}$ :

Lemma 5.2. Let $f \in C^{\infty}\left(\mathbb{T}^{2}\right)$ and $L=\lambda^{\delta}, \theta / 2<\delta<1$. We have

$$
\left|\left\langle f g_{\lambda}, g_{\lambda}\right\rangle-\left\langle f g_{\lambda, L}, g_{\lambda, L}\right\rangle\right| \rightarrow 0
$$

as $\lambda \rightarrow \infty$ along $\Lambda_{g}$.

Proof. Let $f \in C^{\infty}\left(\mathbb{T}^{2}\right)$. We define the multiplication operator $M_{f}: L^{2}\left(\mathbb{T}^{2}\right) \rightarrow$ $L^{2}\left(\mathbb{T}^{2}\right)$ by

$$
M_{f}(g)=f g .
$$

Since $M_{f}$ is a continuous operator on $L^{2}\left(\mathbb{T}^{2}\right)$, we have that

$$
\left\|g_{\lambda}-g_{\lambda, L}\right\|_{2} \rightarrow 0
$$

for $\lambda \in \Lambda_{g}$ implies

$$
\left\|M_{f}\left(g_{\lambda}-g_{\lambda, L}\right)\right\|_{2} \rightarrow 0
$$

and hence

$$
\left|\left\langle M_{f} g_{\lambda}, g_{\lambda}-g_{\lambda, L}\right\rangle\right| \leq\left\|M_{f}\right\|_{\infty}\left\|g_{\lambda}-g_{\lambda, L}\right\| \rightarrow 0 .
$$

If we repeat this, where we switch $g_{\lambda}$ and $g_{\lambda, L}$, we obtain

$$
\left|\left\langle M_{f} g_{\lambda}, g_{\lambda}\right\rangle-\left\langle M_{f} g_{\lambda, L}, g_{\lambda, L}\right\rangle\right| \rightarrow 0 .
$$




\section{Exceptional eigenvalues and a Diophantine inequality}

Fix a nonzero vector $0 \neq \zeta \in \mathcal{L}$, and $\delta \in\left(\frac{\theta}{2}, \frac{1}{2}-\theta\right)$ (such $\delta$ exists because $\theta<1 / 3)$. Let $S_{\zeta}$ be the set of vectors satisfying

$$
S_{\zeta}=\left\{\eta \in \mathcal{L}:|\langle\eta, \zeta\rangle| \leq|\eta|^{2 \delta}\right\}
$$

We define a subset $\Lambda_{\zeta} \subset \Lambda$ of eigenvalues

$$
\Lambda_{\zeta}=\left\{\lambda \in \Lambda: A\left(\lambda, \lambda^{\delta}\right) \cap S_{\zeta}=\emptyset\right\}
$$

(recall that $A(\lambda, L)$ are the lattice points $\eta \in \mathcal{L}$ in the annulus (5.11)). Our goal in this section is to show that

Proposition 6.1. $\Lambda_{\zeta}$ is a subset of density one in $\Lambda$.

Proof. Let

$$
B_{\zeta}=\Lambda \backslash \Lambda_{\zeta}=\left\{\lambda \in \Lambda: A\left(\lambda, \lambda^{\delta}\right) \cap S_{\zeta} \neq \emptyset\right\}
$$

We will show that $B_{\zeta}$ has density zero in the set $\Lambda$ of all perturbed eigenvalues, in fact

$$
\#\left\{\lambda \in B_{\zeta}: \lambda \leq X\right\} \ll \frac{X^{1-\delta^{\prime}}}{|\zeta|}
$$

for $\delta^{\prime}=1 / 2-\theta-\delta>0$.

We first show that

\section{Lemma 6.2.}

$$
\#\left\{\eta \in S_{\zeta}:|\eta|^{2} \leq X\right\} \ll \frac{X^{\frac{1}{2}+\delta}}{|\zeta|}
$$

Proof. Introduce cartesian coordinates with one of the axes in the direction of the vector $\zeta$ by writing every $x \in \mathbb{R}^{2}$ as

$$
x=u \frac{\zeta}{|\zeta|}+v \frac{\zeta^{\perp}}{\left|\zeta^{\perp}\right|}
$$

where if $\zeta=\left(a p, \frac{q}{a}\right)$ then $\zeta^{\perp}=\left(-\frac{q}{a}, p a\right)$ is a vector orthogonal to $\zeta$. In these coordinates,

$$
\langle x, \zeta\rangle=u|\zeta|, \quad|x|^{2}=u^{2}+v^{2}
$$

and our set of lattice points is thus contained in the rectangle

$$
\mathcal{R}=\left\{u \frac{\zeta}{|\zeta|}+v \frac{\zeta^{\perp}}{\left|\zeta^{\perp}\right|}:|u| \leq \frac{X^{\delta}}{|\zeta|}, \quad|v| \leq X^{1 / 2}\right\}
$$

Now estimating the number of lattice points in a rectangle is a simple matter: Putting a fundamental domain $\mathcal{F}=\{(a x, y / a): 0 \leq x, y \leq 1\}$ for the lattice (which has unit area for the case at hand) centered around each lattice point in $\mathcal{R}$, we get a figure whose area is the number of lattice points in question, 
and which is contained in a slightly bigger rectangle whose dimensions are expanded by the diameter $d_{\mathcal{L}}=\sqrt{a^{2}+1 / a^{2}}$ of $\mathcal{F}$ :

$$
\mathcal{R}^{+}=\left\{u \frac{\zeta}{|\zeta|}+v \frac{\zeta^{\perp}}{\left|\zeta^{\perp}\right|}:|u| \leq \frac{X^{\delta}}{|\zeta|}+d_{\mathcal{L}}, \quad|v| \leq X^{1 / 2}+d_{\mathcal{L}}\right\}
$$

Thus we see that $\# \mathcal{L} \cap \mathcal{R}$ is bounded by the area of $\mathcal{R}^{+}$, which is:

$$
\text { area } \mathcal{R}^{+}=2\left(\frac{X^{\delta}}{|\zeta|}+d_{\mathcal{L}}\right) \times 2\left(X^{1 / 2}+d_{\mathcal{L}}\right)=\frac{4 X^{1 / 2+\delta}}{|\zeta|}+O_{\mathcal{L}}\left(X^{1 / 2}\right)
$$

Therefore

$$
\#\left\{\eta \in S_{\zeta}:|\eta|^{2} \leq X\right\} \leq \operatorname{area} \mathcal{R}^{+}=\frac{4 X^{1 / 2+\delta}}{|\zeta|}+O_{\mathcal{L}}\left(X^{1 / 2}\right)
$$

as claimed.

Next we define $\mathcal{N}_{\zeta} \subset \mathcal{N}$ to be the set of norms $|\eta|^{2}$ of $\eta \in S_{\zeta}$, without multiplicities. We clearly have

$$
\#\left\{n \in \mathcal{N}_{\zeta}: n \leq X\right\} \leq \#\left\{\eta \in S_{\zeta}:|\eta|^{2} \leq X\right\} \ll \frac{X^{1 / 2+\delta}}{|\zeta|}
$$

by Lemma 6.2 .

We have a map

$$
\iota: B_{\zeta} \rightarrow \mathcal{N}_{\zeta}
$$

defined by $\iota(\lambda)$ being the closest $n \in \mathcal{N}_{\zeta}$ to $\lambda$; if there are two such elements, i.e. $n_{-}<\lambda<n_{+}$with $n_{ \pm} \in \mathcal{N}_{\zeta}$ and $n_{+}-\lambda=\lambda-n_{-}$, then set $\iota(\lambda)=n_{+}$. Thus we get a well defined map, whose fibers satisfy

$$
\iota^{-1}(n) \subseteq\left\{\lambda \in \Lambda: \exists \eta \in S_{\zeta} \cap A\left(\lambda, \lambda^{\delta}\right),|\eta|^{2}=n\right\} \subseteq \Lambda \cap\left[n-2 n^{\delta}, n+2 n^{\delta}\right]
$$

for $n \gg 1$.

Since $\Lambda$ is interlaced between the norms $\mathcal{N}$, we have

$$
\# \Lambda \cap\left[n-2 n^{\delta}, n+2 n^{\delta}\right] \ll \sum_{n-3 n^{\delta}<m<n+3 n^{\delta}} r_{\mathcal{L}}(m)=\# A\left(n, 3 n^{\delta}\right)
$$

which is the number of lattice points in an annulus. By (2.6),

$$
\# A\left(n, 3 n^{\delta}\right) \ll n^{\delta}+n^{\theta}
$$

and hence (since $\delta<\theta$ )

$$
\# \iota^{-1}(n) \ll n^{\theta}
$$

We thus find

$$
\begin{aligned}
\#\left\{\lambda \in B_{\zeta}: \lambda \leq X\right\} & =\sum_{\substack{n \in \mathcal{N}_{\zeta} \\
n \leq X}} \# \iota^{-1}(n) \\
& \ll X^{\theta} \#\left\{n \in \mathcal{N}_{\zeta}: n \leq X\right\} \ll \frac{X^{1 / 2+\delta+\theta}}{|\zeta|}
\end{aligned}
$$


That is

$$
\#\left\{\lambda \in B_{\zeta}: \lambda \leq X\right\} \ll \frac{X^{1-\delta^{\prime}}}{|\zeta|}
$$

with $\delta^{\prime}=1 / 2-\theta-\delta>0$.

\section{Proof of Theorem 1.1}

7.1. Fixed observables. Fix a nonzero vector $\zeta \in \mathcal{L}$ and recall the definition (6.2), (4.2) of the sets of eigenvalues $\Lambda_{\zeta}$ and $\Lambda_{g}$; both are of density one in $\Lambda$ and thus

$$
\Lambda_{g, \zeta}:=\Lambda_{g} \cap \Lambda_{\zeta}
$$

is still a set of density one in $\Lambda$.

We will show that $\left\langle e_{\zeta} g_{\lambda}, g_{\lambda}\right\rangle \rightarrow 0$ as $\lambda \rightarrow \infty$ along $\Lambda_{g, \zeta}$. By Lemma 5.1 it suffices to show:

Proposition 7.1. Take $\lambda \in \Lambda_{g, \delta}$ and $L=\lambda^{\delta}, \delta \in\left(\frac{\theta}{2}, \frac{1}{2}-\theta\right)$. Fix nonzero $\zeta \in \mathcal{L}$. As $\lambda \rightarrow 0$ while $\lambda \in \Lambda_{g, \zeta}$,

$$
\left\langle e_{\zeta} g_{\lambda, L}, g_{\lambda, L}\right\rangle \rightarrow 0
$$

Proof. For $\xi \in \mathcal{L}$ and $\lambda \in \Lambda_{g, \zeta}$ define

$$
c(\xi)=\frac{1}{|\xi|^{2}-\lambda} .
$$

The $L^{2}$-norm of the truncated Green's function $G_{\lambda, L}\left(L=\lambda^{\delta}\right)$ is given by

$$
\left\|G_{\lambda, L}\right\|_{2}^{2}=\frac{1}{16 \pi^{4}} \sum_{\xi \in A(\lambda, L)} c(\xi)^{2}
$$

and hence

$$
\left\langle e_{\zeta} G_{\lambda, L}, G_{\lambda, L}\right\rangle=\frac{1}{16 \pi^{4}} \sum_{\xi \in A(\lambda, L)} c(\xi) c(\xi-\zeta) .
$$

Cauchy-Schwarz gives

$$
\left|\left\langle e_{\zeta} G_{\lambda, L}, G_{\lambda, L}\right\rangle\right|^{2} \leq\left\|G_{\lambda, L}\right\|_{2}^{2} \sum_{\xi \in A(\lambda, L)} c(\xi-\zeta)^{2} .
$$

Note that for $\lambda \in \Lambda_{\zeta, g}$,

$$
|c(\xi-\zeta)| \ll \frac{1}{L}
$$

Indeed

$$
|\xi-\zeta|^{2}-\lambda=|\xi|^{2}-\lambda-2\langle\xi, \zeta\rangle+|\zeta|^{2}
$$

and since for $\lambda \in \Lambda_{\zeta}$ and $\xi \in A(\lambda, L)$ we have $|\langle\xi, \zeta\rangle|>|\xi|^{2 \delta} \sim L$ we find that

$$
|| \xi-\left.\zeta\right|^{2}-\left.\lambda|\geq 2 L(1+o(1))-L-| \zeta\right|^{2} \gg L
$$


Using (2.6) gives the bound

$$
\sum_{\xi \in A(\lambda, L)} c(\xi-\zeta)^{2} \ll \frac{\# A(\lambda, L)}{L^{2}} \ll \frac{\lambda^{\theta}}{L^{2}}
$$

(recall $\theta \geq 1 / 4$ ) so that we find

$$
\left\langle e_{\zeta} G_{\lambda, L}, G_{\lambda, L}\right\rangle \ll\left\|G_{\lambda}\right\| \frac{\lambda^{\theta / 2}}{L}
$$

The lower bound $\left\|G_{\lambda}\right\| \gg 1 / \lambda^{o(1)}$ of Lemma 4.1 implies

$$
\left\langle e_{\zeta} g_{\lambda, L}, g_{\lambda, L}\right\rangle=\frac{\left\langle e_{\zeta} G_{\lambda, L}, G_{\lambda, L}\right\rangle}{\left\|G_{\lambda, L}\right\|_{2}^{2}} \ll \frac{\lambda^{\theta / 2+o(1)}}{L}=\frac{\lambda^{\theta / 2+o(1)}}{\lambda^{\delta}}
$$

for $\lambda \in \Lambda_{g, \zeta}$, which tends to zero since $\delta>\theta / 2$.

7.2. A diagonalization argument. We have shown that for each $0 \neq \zeta \in$ $\mathcal{L}$, there is a density one subset $\Lambda_{g, \zeta}$ of eigenvalues so that $\left\langle e_{\zeta} g_{\lambda}, g_{\lambda}\right\rangle \rightarrow 0$ as $\lambda \rightarrow \infty$ along $\Lambda_{g, \zeta}$. It remains to see that there is a density one subset $\Lambda_{\infty} \subset \Lambda$ so that for every observable $a \in C^{\infty}\left(\mathbb{T}^{2}\right)$, we have

$$
\left\langle a g_{\lambda}, g_{\lambda}\right\rangle \rightarrow \frac{1}{\operatorname{area}\left(\mathbb{T}^{2}\right)} \int_{\mathbb{T}^{2}} a(x) d x
$$

as $\lambda \rightarrow \infty$ along $\Lambda_{\infty}$. We recall the argument, which can be found e.g. in [7]. For $J \geq 1$, let $\Lambda_{J} \subset \Lambda$ be of density one so that for all $|\zeta| \leq J,\left\langle e_{\zeta} g_{\lambda}, g_{\lambda}\right\rangle \rightarrow 0$ as $\lambda \rightarrow \infty$ along $\Lambda_{g, \zeta}$, and in particular for every trigonometric polynomial $P_{J}(x)=\sum_{|\zeta| \leq J} p_{\zeta} e_{\zeta}(x)$ we have

$$
\left\langle P_{J} g_{\lambda}, g_{\lambda}\right\rangle \rightarrow \frac{1}{\operatorname{area}\left(\mathbb{T}^{2}\right)} \int_{\mathbb{T}^{2}} P_{J}(x) d x
$$

We may assume that $\Lambda_{J+1} \subseteq \Lambda_{J}$ for each $J$. Now choose $M_{J}$ so that for all $X>M_{J}$

$$
\frac{1}{\#\{\lambda \in \Lambda: \lambda \leq X\}} \#\left\{\lambda \in \Lambda_{J}: \lambda \leq X\right\} \geq 1-\frac{1}{2^{J}}
$$

and let $\Lambda_{\infty}$ be such that $\Lambda_{\infty} \cap\left[M_{J}, M_{J+1}\right]=\Lambda_{J} \cap\left[M_{J}, M_{J+1}\right]$ for all $J$. Then $\Lambda_{\infty} \cap\left[0, M_{J+1}\right]$ contains $\Lambda_{J} \cap\left[0, M_{J+1}\right]$ and therefore $\Lambda_{\infty}$ has density one in $\Lambda$ and (7.10) holds for $\lambda \in \Lambda_{\infty}$. Since the trigonometric polynomials are dense in $C^{\infty}\left(\mathbb{T}^{2}\right)$ in the uniform norm and the probability measures $\left|g_{\lambda}(x)\right|^{2} d x$ are continuous with respect to this norm, we find that (7.9) holds.

\section{Appendix A. A Rigorous Description of the Point SCATterer}

Denote by $\delta_{x_{0}}$ the Dirac distribution at $x_{0} \in \mathbb{T}^{2}$. We are interested in solutions to the equation

$$
(\Delta+\lambda) f=\delta_{x_{0}}, \quad f \in C^{\infty}\left(\mathbb{T}^{2} \backslash\left\{x_{0}\right\}\right),\|f\|_{2}=1
$$


and its association with the eigenfunctions and eigenvalues of a family of self-adjoint operators. Consider the domain of $C^{\infty}$-functions which vanish in a neighborhood of $x_{0}$

$$
D_{0}=C_{0}^{\infty}\left(\mathbb{T}^{2} \backslash\left\{x_{0}\right\}\right)
$$

and denote $-\Delta_{x_{0}}=-\left.\Delta\right|_{D_{0}}$. The operators associated with equation (A.1) form the family of self-adjoint extensions of the positive symmetric operator $-\Delta_{x_{0}}$ (cf. [6], section 1, p. 277).

We make the following conventions in the definition of the Green's function.

Definition 1. Denote by $\sigma(-\Delta)$ the spectrum of $-\Delta$ on $C^{2}\left(\mathbb{T}^{2}\right)$. We define the Green's function $G_{\lambda}(x ; y)$ at energy $\lambda \in \mathbb{C} \backslash \sigma(-\Delta)$ on $\mathbb{T}^{2}$ as the integral kernel of the resolvent $(\Delta+\lambda)^{-1}$, that is

$$
(\Delta+\lambda)^{-1} f(y)=\int_{\mathbb{T}^{2}} G_{\lambda}(x, y) f(x) d x .
$$

In order to give a self-contained presentation of the theory of self-adjoint extensions, we briefly recall the standard definitions of the adjoint of an operator, symmetry and self-adjointness.

Definition 2. Let $H$ be a Hilbert space and $\operatorname{Dom}(B) \subset H$. Consider the operator $B: \operatorname{Dom}(B) \rightarrow H$. We define

$$
\operatorname{Dom}\left(B^{*}\right)=\{y \in H \mid \exists a \in H: \forall x \in \operatorname{Dom}(B):\langle B x, y\rangle=\langle x, a\rangle\}
$$

then we define the adjoint $B^{*}$ of $B$ as the map $B^{*}: \operatorname{Dom}\left(B^{*}\right) \rightarrow H$,

$$
B^{*} y=a \text {. }
$$

We call $B$ symmetric if

$$
\forall x, y \in \operatorname{Dom}(B):\langle B x, y\rangle=\langle x, B y\rangle .
$$

We call $B$ self-adjoint if $B$ is symmetric and

$$
\operatorname{Dom}(B)=\operatorname{Dom}\left(B^{*}\right) \text {. }
$$

We have the following well-known results from self-adjoint extension theory which we summarize briefly. Proofs can be found in [18], Chapter X.1. We will give the relevant references for each lemma.

Definition 3. Let $B$ be a densely defined symmetric operator on a Hilbert space $H$. Denote its adjoint by $B^{*}$. Let $\eta \in \mathbb{C} \backslash \mathbb{R}$. The deficiency spaces of $B$ at $\eta$ and $\bar{\eta}$ are defined as

$$
D_{\eta}(B)=\operatorname{ker}\left\{B^{*}-\eta\right\}, \quad D_{\bar{\eta}}(B)=\operatorname{ker}\left\{B^{*}-\bar{\eta}\right\} .
$$

We refer to the members of a basis of a deficiency space as deficiency elements.

The following lemma is proven as part of Theorem X.1, p. 136 in [18].

Lemma A.1. As a function of $\eta, \operatorname{dim} D_{\eta}(B)$ is constant on the upper (lower) complex half-plane. 
We proceed with the definition of the deficiency indices of a closed symmetric operator which indicate if the operator can be extended to a selfadjoint operator.

Definition 4. If $\operatorname{dim} D_{\eta}(B)=m$ and $\operatorname{dim} D_{\bar{\eta}}(B)=n$ for nonnegative integers $m, n$, we say that the operator $B$ has deficiency indices $(m, n)$.

The following lemma is Corollary (a), p. 141 in [18].

Lemma A.2. $B$ has deficiency indices $(0,0)$ if, and only if, $B$ is self-adjoint.

If the deficiency indices are nonzero and equal, then a family of self-adjoint extensions exists and can be constructed as follows. This lemma combines Theorem X.2, p. 140 and Corollary (b), p. 141 in [18.

Lemma A.3. If a closed positive symmetric operator $B$ has deficiency indices $(n, n), n \geq 1$, then for each unitary map $U: D_{\mathrm{i}}(B) \rightarrow D_{-\mathrm{i}}(B)$ there is a self-adjoint extension $B_{U}: D_{U} \rightarrow H$, where

$$
D_{U}=\left\{f=g+h+U h \mid(g, h) \in \operatorname{Dom}(B) \times D_{\mathrm{i}}(B)\right\}
$$

and $B_{U}$ acts as follows

$$
B_{U} f=B g+\mathrm{i} h-\mathrm{i} U h .
$$

The operator $B_{U}$ has deficiency indices $(0,0)$. Conversely, every self-adjoint extension of $B$ is of this form.

We apply Lemma A.3 to construct a one parameter family of self-adjoint extensions of the operator $-\Delta_{x_{0}}$. Denote the domain of the closure of $-\Delta_{x_{0}}$ by $\tilde{D}_{0}$.

Lemma A.4. The operator $-\Delta_{x_{0}}$ has deficiency indices $(1,1)$. The corresponding deficiency elements are the Green's functions $G_{\mathrm{i}}\left(x, x_{0}\right), G_{-\mathrm{i}}\left(x, x_{0}\right)$.

The self-adjoint extensions of $-\Delta_{x_{0}}$ are given by the one parameter family

$$
-\Delta_{\varphi}: D_{\varphi} \rightarrow L^{2}\left(\mathbb{T}^{2}\right), \quad \varphi \in(-\pi, \pi]
$$

where

$$
D_{\varphi}=\left\{g+c G_{\mathrm{i}}+c e^{\mathrm{i} \varphi} G_{-\mathrm{i}}: g \in \tilde{D}_{0}, c \in \mathbb{C}\right\}
$$

and

$$
-\Delta_{\varphi} f=-\Delta g+c \mathrm{i} G_{\mathrm{i}}-c e^{\mathrm{i} \varphi \mathrm{i} G_{-\mathrm{i}}} .
$$

Proof. By definition of the Green's function we have that

$$
\operatorname{ker}\left\{\left(\Delta_{x_{0}}\right)^{*} \pm \mathrm{i}\right\}=\mathcal{L}\left\{G_{ \pm \mathrm{i}}\right\} .
$$

Hence $-\Delta_{x_{0}}$ has deficiency indices $(1,1)$ and we may apply Lemma A.3 to obtain the result.

Remark A.5. Note that $-\Delta_{\pi}$ recovers the Laplacian on $C^{\infty}\left(\mathbb{T}^{2}\right)$.

Next we derive an equation for eigenvalues of the operator $-\Delta_{\varphi}, \varphi \in$ $(-\pi, \pi)$. 
Lemma A.6. Let $\varphi \in(-\pi, \pi)$. We have that $\lambda \notin \sigma(-\Delta)$ is an eigenvalue of $-\Delta_{\varphi}$ if, and only if,

$$
\sum_{\xi \in \mathcal{L}}\left(\frac{1}{|\xi|^{2}-\lambda}-\frac{|\xi|^{2}}{|\xi|^{4}+1}\right)=c_{0} \tan (\varphi / 2)
$$

where

$$
c_{0}=\sum_{\xi \in \mathcal{L}} \frac{1}{|\xi|^{4}+1} .
$$

The corresponding eigenfunction is a multiple of $G_{\lambda}\left(x ; x_{0}\right)$.

Proof. Let $f \in D_{\varphi}$ and $\|f\|_{2}=1$. Then $f$ must be of the form

$$
f=g+c G_{\mathrm{i}}+c e^{\mathrm{i} \varphi} G_{-\mathrm{i}}, \quad g \in D_{0}, c \in \mathbb{C} .
$$

Let us first assume that $\lambda \notin \sigma(-\Delta)$ is an eigenvalue of $-\Delta_{\varphi}$. We have

$$
0=\left(\Delta_{\varphi}+\lambda\right) f=(\Delta+\lambda) g+c(\lambda-\mathrm{i}) G_{\mathrm{i}}+c e^{\mathrm{i} \varphi}(\lambda+\mathrm{i}) G_{-\mathrm{i}} .
$$

We apply the resolvent $(\Delta+\lambda)^{-1}$ to both sides to obtain

$$
0=g+c \frac{\lambda-\mathrm{i}}{\Delta+\lambda} G_{\mathrm{i}}+c e^{\mathrm{i} \varphi} \frac{\lambda+\mathrm{i}}{\Delta+\lambda} G_{-\mathrm{i}} .
$$

In view of the iterated resolvent identity

$$
(\lambda \mp \mathrm{i}) \frac{1}{\Delta+\lambda} \frac{1}{\Delta \pm \mathrm{i}}=\frac{1}{\Delta \pm \mathrm{i}}-\frac{1}{\Delta+\lambda}
$$

we can rewrite equation (A.13) as

$$
0=g(x)+c\left(G_{\mathrm{i}}-G_{\lambda}\right)\left(x, x_{0}\right)+c e^{\mathrm{i} \varphi}\left(G_{-\mathrm{i}}-G_{\lambda}\right)\left(x, x_{0}\right) .
$$

In particular

$$
f=g+c\left(G_{\mathrm{i}}+e^{\mathrm{i} \phi} G_{-\mathrm{i}}\right)=c\left(1+e^{\mathrm{i} \phi}\right) G_{\lambda}
$$

and so $f$ is a multiple of $G_{\lambda}$.

If we now take the limit $x \rightarrow x_{0}$ on the r.h.s. of (A.15) we obtain

$$
0=\lim _{x \rightarrow x_{0}}\left(G_{\mathrm{i}}-G_{\lambda}\right)\left(x, x_{0}\right)+e^{\mathrm{i} \varphi} \lim _{x \rightarrow x_{0}}\left(G_{-\mathrm{i}}-G_{\lambda}\right)\left(x, x_{0}\right)
$$

and note that $\lambda \notin \sigma(-\Delta)$ implies $c \neq 0$ so we may drop the constant. A simple rearrangement of this equation yields

$$
\tan (\varphi / 2) \lim _{x \rightarrow x_{0}} \operatorname{Im} G_{\mathrm{i}}\left(x, x_{0}\right)=\lim _{x \rightarrow x_{0}}\left(G_{\lambda}-\operatorname{Re} G_{\mathrm{i}}\right)\left(x, x_{0}\right) .
$$

In order to obtain equation (A.10) we require the following $L^{2}$-identity for the Green's function $G_{\lambda}$ on $\mathbb{T}^{2}$

$$
G_{\lambda}\left(x, x_{0}\right)=-\frac{1}{4 \pi^{2}} \sum_{\xi \in \mathcal{L}} \frac{\exp \left(\mathrm{i} \xi \cdot\left(x-x_{0}\right)\right)}{|\xi|^{2}-\lambda}, \quad x \neq x_{0}
$$


We rewrite the r.h.s. of (A.18) as

$$
\begin{aligned}
& -\frac{1}{4 \pi^{2}} \sum_{\xi \in \mathcal{L}} e\left(\xi \cdot\left(x-x_{0}\right)\right)\left\{\frac{1}{|\xi|^{2}-\lambda}-\operatorname{Re} \frac{1}{|\xi|^{2}-\mathrm{i}}\right\} \\
= & -\frac{1}{4 \pi^{2}} \sum_{\xi \in \mathcal{L}} e\left(\xi \cdot\left(x-x_{0}\right)\right)\left\{\frac{1}{|\xi|^{2}-\lambda}-\frac{|\xi|^{2}}{|\xi|^{4}+1}\right\} \\
& \stackrel{x \rightarrow x_{0}}{\longrightarrow}-\frac{1}{4 \pi^{2}} \sum_{\xi \in \mathcal{L}}\left\{\frac{1}{|\xi|^{2}-\lambda}-\frac{|\xi|^{2}}{|\xi|^{4}+1}\right\} .
\end{aligned}
$$

Finally, note that

$$
\lim _{x \rightarrow x_{0}} \operatorname{Im} G_{\mathrm{i}}\left(x, x_{0}\right)=-\frac{1}{4 \pi^{2}} \sum_{\xi \in \mathcal{L}} \frac{1}{|\xi|^{4}+1} .
$$

To see the reverse implication assume that $\lambda$ solves equation (A.17), a rearrangement of equation (A.10). The r.h.s. of equation (A.17) has singularities at points which are in $\sigma(-\Delta)$, hence $\lambda \notin \sigma(-\Delta)$. We define

$$
f_{\lambda}(x)=\left(G_{\lambda}-G_{\mathrm{i}}\right)\left(x, x_{0}\right)+e^{\mathrm{i} \varphi}\left(G_{\lambda}-G_{-\mathrm{i}}\right)\left(x, x_{0}\right)
$$

and observe that

$$
\left(1+e^{\mathrm{i} \varphi}\right) G_{\lambda}=f_{\lambda}+G_{\mathrm{i}}+e^{\mathrm{i} \varphi} G_{-\mathrm{i}} \in D_{\varphi}
$$

because equation (A.17) implies $f_{\lambda}\left(x_{0}\right)=0$. The iterated resolvent identity (A.14) implies

$$
\left(\Delta_{\varphi}+\lambda\right) f_{\lambda}=(\Delta+\lambda) f_{\lambda}=-(\lambda-\mathrm{i}) G_{\mathrm{i}}-(\lambda+\mathrm{i}) G_{\mathrm{i}}
$$

and by the definition of the operator $\Delta_{\varphi}$ we have

$$
\left(1+e^{\mathrm{i} \varphi}\right)\left(\Delta_{\varphi}+\lambda\right) G_{\lambda}=(\Delta+\lambda) f_{\lambda}+(\lambda-\mathrm{i}) G_{\mathrm{i}}+(\lambda+\mathrm{i}) G_{-\mathrm{i}}=0 .
$$

This concludes the proof.

\section{REFERENCES}

[1] R. P. Bambah and S. Chowla. On numbers which can be expressed as a sum of two squares. Proc. Nat. Inst. Sci. India, 13, 101-103 (1947).

[2] G. Berkolaiko, J. P. Keating and B. Winn. Intermediate wave functions statistics, Phys. Rev. Lett. 91, 134103 (2003).

[3] E. Bogomolny, U. Gerland and C. Schmit. Singular statistics. Phys. Rev. E (3) 63 (2001), no. 3, part 2, 036206.

[4] E. Bogomolny, O. Giraud and C. Schmit. Nearest-neighbor distribution for singular billiards. Phys. Rev. E (3) 65 (2002), no. 5, 056214.

[5] T. Shigehara and Taksu Cheon, Wave chaos in quantum billiards with a small but finite-size scatterer. Phys. Rev. E 54, 1321-1331 (1996).

[6] Y. Colin de Verdière. Pseudo-laplaciens I. Annales de l'Institut Fourier, tome 32, no. 3, 275-286, 1982.

[7] Y. Colin de Verdière Ergodicité et fonctions propres du laplacien. Comm. Math. Phys. 102 (1985), no. 3, 497-502. 
[8] D. A. Hejhal. Some observations concerning eigenvalues of the Laplacian and Dirichlet L-series. Recent progress in analytic number theory, Symp. Durham 1979, Vol. 2, 95110 (1981).

[9] M. N Huxley, Exponential sums and lattice points. III. Proc. London Math. Soc. (3) 87 (2003), no. 3, 591-609.

[10] D. Jakobson. Quantum limits on flat tori. Ann. of Math. (2) 145 (1997), 235-266.

[11] J. P. Keating, J. Marklof and B. Winn. Localized eigenfunctions in S̆eba billiards. J. Math. Phys. 51 (2010), no. 6, 062101.

[12] S. Kerckhoff, H. Masur and J. Smillie, Ergodicity of billiard flows and quadratic differentials. Ann. of Math. (2) 124 (1986), no. 2, 293-311.

[13] R. de L. Kronig and W. G. Penney, Quantum Mechanics of Electrons in Crystal Lattices. Proceedings of the Royal Society of London. Series A, Vol. 130, No. 814 (Feb. 3, 1931), pp. 499-513.

[14] E. Landau, Über die Einteilung der positiven ganzen Zahlen in vier Klassen nach der Mindeszahl der zu ihrer additiven Zusammensetzung erforderlichen Quadrate. Arch. Math. Phys. 13, 305-312, 1908.

[15] J. Marklof and Z. Rudnick, Almost all eigenfunctions of a rational polygon are uniformly distributed. J. of Spectral Theory. 2 (2012), 107-113.

[16] F. Oravecz, Z. Rudnick and I. Wigman, The Leray measure of nodal sets for random eigenfunctions on the torus, Annales de l'Institut Fourier 58 (1), (2008), 299-335.

[17] S. Rahav and S. Fishman. Spectral statistics of rectangular billiards with localized perturbations. Nonlinearity 15 (2002), no. 5, 1541-1594.

[18] M. Reed and B. Simon. Methods of Modern Mathematical Physics, Vol. 2: Fourier Analysis and Self-Adjointness. Academic Press, London, 1975.

[19] P. S̆eba. Wave Chaos in Singular Quantum Billiard. Phys. Rev. Letters, Vol. 64, No. 16, 1855-58, 1990.

[20] T. Shigehara, Conditions for the appearance of wave chaos in quantum singular systems with a pointlike scatterer. Phys. Rev. E 50, 4357-4370 (1994).

[21] A. Snirel'man Ergodic properties of eigenfunctions. Uspekhi Mat. Nauk 29, no. 6 (180), 181-182, 1974

[22] J. G . van der Corput, Neue zahlentheoretische Abschätzungen. Math. Ann. 89 (1923), no. 3-4, 215-254.

[23] S. Zelditch. Uniform distribution of eigenfunctions on compact hyperbolic surfaces. Duke Math. J. 55, no. 4, 919-941, 1987.

[24] S. Zelditch and M. Zworski, Ergodicity of eigenfunctions for ergodic billiards. Comm. Math. Phys. 175, 673-682, (1996).

Raymond and Beverly Sackler School of Mathematical Sciences, Tel Aviv University, Tel Aviv 69978, IsRael

E-mail address: rudnick@post.tau.ac.il

Raymond and Beverly Sackler School of Mathematical Sciences, Tel Aviv University, Tel AVIV 69978, IsRael

E-mail address: henrik@post.tau.ac.il 